\title{
Experiencia Afectiva Usuario en ambientes con Inteligencia Artificial, Sensores Biométricos y/o Recursos Digitales Accesibles: Una Revisión Sistemática de Literatura
}

Johana Miroslava Fernández-Ordóñez ${ }^{1}$, Ligia Elena Maza Jiménez ${ }^{1}$, Pablo TorresCarrión ${ }^{1}$, Luis Barba-Guamán, Germania Rodríguez-Morales ${ }^{1}$

jmfernandez1@utpl.edu.ec, lemaza@utpl.edu.ec, pvtorres@utpl.edu.ec, lrbarba@utpl.edu.ec, grrodriguez@utpl.edu.ec

${ }^{1}$ Universidad Técnica Particular de Loja, San Cayetano Alto 1101608, Loja, Ecuador

DOI: 10.17013/risti.35.35-53

Resumen: Proponemos una revisión sistemática de literatura que considera la Experiencia Afectiva de Usuario (UAX) en ambientes con Inteligencia Artificial (IA), Sensores Biométricos y/o Recursos Digitales accesibles, haciendo singular enfoque en las adaptaciones para alumnos con Síndrome de Down. Se aplica el método de Torres-Carrión, planteando cinco preguntas de investigación: a) estándares de UX en entornos educativos, b) estrategias aplicadas con sensores biométricos para evaluar UAX, c) métodos/instrumentos para evaluar UAX de personas con Síndrome de Down en ambientes educativos, d) características de los recursos didácticos desarrollados para personas con discapacidades cognitivas y e) estrategias utilizadas en investigaciones de UAX e IA. La búsqueda en la base de datos Scopus genera 17 artículos y 37 revistas. Como resultados destacan los juegos y robótica como principales recursos para estimular el aprendizaje en personas con discapacidad cognitiva, así como, los métodos de indagación con resultados relevantes y eficientes, utilizando sensores fisiológicos y ambientales.

Palabras-clave: Experiencia Afectiva de Usuario, Inteligencia Artificial, Sensores biométricos, Recursos Didácticos accesibles, Síndrome de Down.

User Affective Experience in environments with Artificial Intelligence, Biometric Sensors and/or Accessible Digital resources. A Systematic Literature Review

Abstract: We propose a systematic review of literature that considers the User Affective Experience (UAX) in environments with Artificial Intelligence (AI), Biometric Sensors and/or accessible Digital Resources, making a singular focus on adaptations for students with Down Syndrome. The Torres-Carrión method is applied, raising five research questions: a) UX standards in educational environments, b) strategies applied with biometric sensors to evaluate UAX, c) methods / instruments to evaluate UAX of people with Down Syndrome in 
educational environments, d) characteristics of the didactic resources developed for people with cognitive disabilities and e) strategies used in UAX and AI research. The search in the Scopus database generates 17 articles and 37 journals. As results, games and robotics stand out as the main resources to stimulate learning in people with cognitive disabilities, as well as the methods of inquiry with relevant and efficient results, using physiological and environmental sensors.

Keywords: User Affective Experience, Artificial Intelligence, Biometric Sensors, Accessible Digital resources, Down Syndrome.

\section{Introducción}

La Interacción Humano-Computador (IHC) es el área emergente de la informática más cercana al usuario, que estudia la comunicación que se produce entre los ordenadores y las personas que los utilizan (Marcos, 2001; Sears \& Jacko, 2007). En este contexto la Experiencia de Usuario (UX) se refiere al sentir de la persona cuando interactúa con un sistema informático (Boada, 2017) y maneja principalmente tres dimensiones o experiencias: la estética, la significativa y la afectiva; ésta última está orientada a medir la experiencia final del usuario por medio de diferentes tipos de emociones como: diversión, ira, desprecio, satisfacción, asco, emoción, miedo, culpa, orgullo por el logro, alivio, tristeza / angustia, placer sensorial y vergüenza (Ekman, Paul \& Friesen, 1971). La emociones despiertan los recuerdos que perduran y la capacidad de enganche emocional que se puede producir, convirtiéndose de esta manera en la Experiencia Afectiva de Usuario (UAX) (Buck, Khan, Fagan, \& Coman, 2018)LLC We measured reported User Affective Experience (UAX. En este ámbito emergente de la ciencia, se propone una investigación bibliográfica, que permita sostener futuras propuestas de investigación.

La investigación en UAX, al igual que otras ciencias, se ha visto enriquecida por la Inteligencia Artificial(IA), que ha permitido establecer entornos deinteracción inteligente que percibe las emociones del usuario (Mira, 1999). Según García A. (2012), la IA trata de explicar el funcionamiento mental basándose en el desarrollo de algoritmos para controlar diferentes cosas, combinando varios campos, como la robótica, los sistemas expertos y otros, los cuales tienen un mismo objetivo, que es tratar de crear máquinas que puedan pensar por sí solas. La IA puede dividirse en tres amplios escenarios (Gomes \& Preto, 2018, p. 64): a) estrecha o débil que se limita a un área funcional; b) general, que comprende el poder del razonamiento, la resolución de problemas y el pensamiento abstracto; $y, c)$ la súper inteligencia que es el nivel máximo cuando la IA supera la inteligencia humana. Dentro de la IA débil se encuentra la Conversacional, con una fuerte relación con IHC, misma que garantiza que se pueda realizar conversaciones simuladas con una computadora (Brinquis, 2019), abriendo el espacio para desarrollar comportamientos empáticos entre la máquina y el usuario, siendo un gran aporte en el desarrollo de UAX.

Acorde a lo anterior, IHC ha evolucionado apoyado en IA, visible en ambientes inteligentes con soporte en IoT. Para mejorar la interacción, como nuevos instrumentos que ayudan a transformar un rasgo físico y concreto de un ser humano en una señal eléctrica, han surgido los Sensores Biométricos, que tienen capacidades para medir, codificar, comparar, almacenar, transmitir y/o reconocer características propias de una persona utilizando diferentes tipos de sistemas biométricos tales como reconocimiento 
de la huella dactilar, cara, iris/retina, geometría de dedos/mano, autentificación de la voz y reconocimiento de la firma (Tolosa \& Giz, 2010). Estos sensores permiten la comunicación entre el mundo real y digital, ya que mientras más datos se reciban sobre las distintas emociones que se pueden sentir, más precisa será la tecnología.

Los ambientes inteligentes están transformando la metodología de enseñanza aprendizaje en espacios interactivos, permitiendo conocer las percepciones de los involucrados, su interelación con los demás y el entorno; sus habilidades motrices, con las cuales se facilita la interacción y con ello el aprendizaje; mejores momentos para la estimulación y para el descanso; todo esto como un aporte innovador en la ingeniería de la enseñanza. En estos ambientes enriquecidos, el diseño de recursos didácticos digitales, desde estándares de accesibilidad ha permitido que personas con discapacidades y limitantes de aprendizaje, tales como las personas con Síndrome de Down, tengan la oportunidad de fortalecer los procesos psicológicos básicos como percepción (Herrero Nivela, Vived Conte, \& Digitalia, 2007), memoria (Florez, 2004), lenguaje, comunicación (Rondal, Perera, \& Nadel, 2000) y atención (Castells \& Wisniewski, 1994). Estas áreas, por su interés, son consideradas para el estudio, en una de las preguntas de investigación.

Para el desarrollo de esta revisión de literatura se sigue el método propuesto por TorresCarrión (2018)as well as for justifying future research in said area. This process is complex for beginners in scientific research, especially if you have not developed skills for searching and filtering information, and do not know which high-level databases are relevant in their field of study. The method proposed leads the researcher from 'My' to 'The' current state of the problem; we propose an adaptation of the method by Kitchenham and Bacca, which divides the process into three sub-parts: planning, conducting and reporting results. From the approach of the research problem in the preliminary phase research questions (recommended between 3 to 5 detallado en la segunda sección; se proponen cinco preguntas de investigación que incluyen: estándares que describen la UX en entornos educativos inclusivos, estrategias aplicadas con sensores biométricos para evaluar UAX, métodos e instrumentos considerados en la UAX, recursos y estrategias didácticas que unifican UAX e IA. Para dar respuestas, en el proceso de búsqueda, se obtienen 338 artículos, que se redujeron a 44 luego de aplicar criterios y protocolos de inclusión y exclusión, y finalmente 17 al aplicar criterios de calidad.

\section{Metodología}

Para el SLR, se aplica la Metodología de Torres-Carrión (2018)as well as for justifying future research in said area. This process is complex for beginners in scientific research, especially if you have not developed skills for searching and filtering information, and do not know which high-level databases are relevant in their field of study. The method proposed leads the researcher from 'My' to 'The' current state of the problem; we propose an adaptation of the method by Kitchenham and Bacca, which divides the process into three sub-parts: planning, conducting and reporting results. From the approach of the research problem in the preliminary phase research questions (recommended between 3 to 5, que contempla tres fases acorde a la propuesta de Kitchenham (2009): a) Planificación, b) Reporte de la revisión y c) Presentación de resultados. La fase de planificación, inicia con el conocimiento detallado del ámbito teórico en el cual se va a aportar, explicado en un mentefacto conceptual (ver Figura 1), base para establecer 
el tesauro científico a utilizarse en la estructura semántica de búsqueda (Ver Tabla 2); además, como guía para el estudio, se proponen cinco preguntas de investigación. Desde estos dos insumos, se desarrollan todos los elementos de las fases de reporte de la revisión y presentación de resultados (Ver Tabla 1).

\begin{tabular}{lll}
\hline Planificación & Reporte de la revisión & $\begin{array}{l}\text { Presentación de } \\
\text { resultados }\end{array}$ \\
\hline Mentefacto Conceptual & Identificación de la Búsqueda & $\begin{array}{l}\text { Resultados } \\
\text { Conclusiones }\end{array}$ \\
Estructura semántica de búsqueda & Selección de estudios primarios & \\
$\begin{array}{l}\text { Preguntas de investigación } \\
\text { Revisiones de Protocolos de Revisión } \\
\text { Selección de revistacas Relacionadas y Base de datos }\end{array}$ & $\begin{array}{l}\text { Evaluación de la calidad del estudio } \\
\text { Extracción y seguimiento de datos }\end{array}$ & \\
\hline
\end{tabular}

Tabla 1 - Fases de la Metodología Torres-Carrión

\subsection{Constructos teóricos}

Un constructo es una construcción teórica para comprender un problema determinado y según la metodología aplicada en el estudio, se organiza a través del mentefacto conceptual, que "permite al Investigador centrar su atención en el contexto teórico real de la investigación” (Torres-Carrión, González-González, Bernal-Bravo, \& InfanteMoro, 2019).

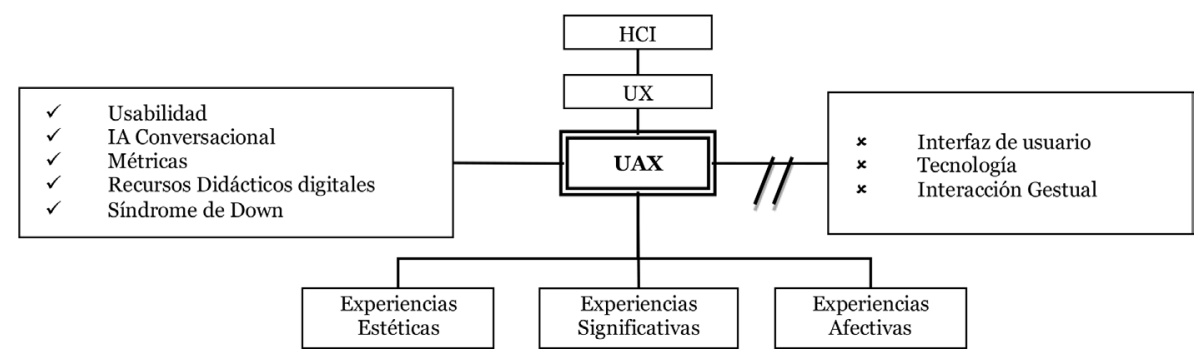

Figura 1 - Mentefacto Conceptual de Experiencia Afectiva de Usuario

El mentefacto expuesto en la Figura 1, detalla la estructura conceptual de la UAX; concepto que nace desde las áreas de la IHC y UX. Como ámbitos o subclases según (Desmet \& Hekkert, 2007) destacan la experiencia estética, significativa y afectiva. Otros campos de la ciencia, que son parte de UX, empero, que no serán estudiados son: interfaz de usuario, tecnología e interacción gestual. Finalmente, las características de UAX que son de interés para nuestro estudio están visibles en la parte izquierda del mentefacto: usabilidad, IA conversacional, métricas de UAX y recursos didácticos digitales inclusivos estudiados para personas con Síndrome de Down.

\subsection{Desarrollo de Protocolos de Revisión}

Se definió criterios generales, de exclusión y de calidad, relacionados con las preguntas planteadas. 


\subsubsection{Criterios Generales}

- $\quad$ Estudios que involucran UAX e IA.

- $\quad$ Publicaciones de los cinco últimos años, es decir, desde el año 2015 al 2019.

- $\quad$ La Base de Datos utilizada en la presente investigación es Scopus.

\subsubsection{Criterios de Exclusión}

- No se consideran como Experiencia de Usuario: La interfaz de usuario, la tecnología, ni la Interacción Gestual.

- No se contempló revistas que no están catalogadas como artículos científicos: editoriales, reseñas de libros, informes técnicos, etc.

- Se consideraron únicamente publicaciones referentes a Ciencias de la Computación, Ciencias Sociales y Psicología.

\subsubsection{Criterios de calidad}

Para la búsqueda de los estándares que describen la UX que son aplicados en entornos educativos inclusivos requeridos en la RQ1, se consideró la UX, la Usabilidad, el Diseño centrado en el ser humano para sistemas interactivos, así como la estructura del Sistema de Educación y los tipos de discapacidades.

Para saber que estrategias se han aplicado con sensores biométricos para evaluar UAX según la RQ2, se han utilizado variables como tipo de sensores, métricas y tipo de análisis.

En lo que se refiere a los métodos e instrumentos planteados en la RQ3, se consideró la UAX, emociones, educación; así como el Síndrome de Down, en sus niveles y limitaciones conforme sus capacidades intelectuales y cognitivas.

Con respecto de la RQ4, se consideraron publicaciones referentes a Recursos Didácticos digitales con su respectiva clasificación y categorías de evaluación enfocándonos en discapacidad cognitiva.

Finalmente, es necesario conocer si existen investigaciones conjuntas de Experiencia Afectiva de Usuario con Inteligencia Artificial, a fin de verificar si se puede hacer uso de alguna tecnología que permita evaluar las emociones de los usuarios, conforme lo planteado en la RQ5.

\subsection{Estructura semántica de búsqueda}

Desde el mentefacto conceptual, se elabora la estructura semántica (Tabla 2), sostenida en la sinonimia y el tesauro científico (“Tesauro científico,” 2019). Para la obtención del tesauro, como herramienta de búsqueda se utiliza la página web (Thesaurus.com, 2019), que muestra en una escala visual la similitud de los conceptos, así como un detalle de su significado. Para la elección de los sinónimos, se realiza por sugerencia de un experto en el área del conocimiento, y en base a la relación que pueda existir con el ámbito del problema propuesto.

La búsqueda se organiza en tres niveles: UAX (L1), IA (L2) y la aplicación de cuatro Protocolos de Revisión (L3). Existe un nivel intermedio en el cual se aplica la unión de 
los resultados del L1 y L2, obteniendo en la intersección de estos dos grandes conjuntos, los artículos que refieren a UAX e IA $(n=338)$.

\begin{tabular}{|c|c|c|c|c|}
\hline \multirow[b]{2}{*}{ L1 } & \multirow[b]{2}{*}{ UAX } & $\begin{array}{l}\text { USER } \\
\text { EXPERIENCE }\end{array}$ & $\begin{array}{l}\text { (ux OR cx OR ((user* OR customer OR person } \\
\text { OR people OR child* OR quality OR design OR } \\
\left.\text { conversat* }^{*} \mathrm{~W} / 2(\text { experienc* OR satisfaction })\right) \text { ) }\end{array}$ & 219,790 \\
\hline & & AFFECTIVE & $\begin{array}{l}\text { W/4 ( ( affectiv* OR emoti* OR sentiment* OR } \\
\text { feel* OR sens* OR perceptual OR visceral ) OR } \\
\text { ( intuitive OR surprisse OR excit* OR passionate } \\
\text { OR pleasant) OR ( anxiety OR eagerness OR } \\
\text { angst OR nervousness OR concern OR doubt } \\
\text { OR dread OR jitters OR panic OR suspense OR } \\
\text { trouble OR uneasiness OR uncertainty ) OR ( } \\
\text { happ* OR joy* OR cheerfulness OR gladness } \\
\text { OR gaiety OR playfulness OR contentement OR } \\
\text { rejoicing OR fun OR merriment OR mirth ) OR } \\
\text { ( angry OR disgust OR displeasure OR distaste } \\
\text { OR chagrin ) OR ( dissatisfaction OR trouble OR } \\
\text { disinterest ) OR ( sadness OR sorrow OR gloom } \\
\text { OR misery OR dreariness OR melancholia ) OR } \\
\text { ( anger OR wrath OR ire OR rage OR temper ) } \\
\text { OR ( fear OR trepidation OR apprehension OR } \\
\text { nervousness OR frightened ) ) ) }\end{array}$ & 7,318 \\
\hline L2 & IA & $\begin{array}{l}\text { ARTIFICIAL } \\
\text { INTELLIGENCE }\end{array}$ & 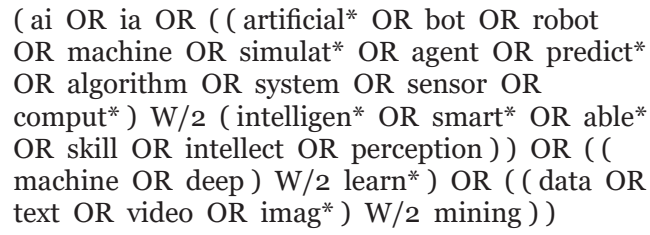 & $1,089,667$ \\
\hline $\begin{array}{l}{[\mathrm{L} 1 \mathrm{AND}} \\
\mathrm{L} 2]\end{array}$ & \multicolumn{2}{|c|}{ [UAX AND IA] } & & 338 \\
\hline \multirow{6}{*}{ L3 } & \multicolumn{3}{|c|}{ PROTOCOLS } & \\
\hline & \multicolumn{2}{|l|}{ Year } & $2015-2019$ & 203 \\
\hline & \multicolumn{2}{|c|}{ Subject área } & Computer Science, Social Sciences, Psychology & 166 \\
\hline & \multicolumn{2}{|c|}{ Document Type } & Article & 45 \\
\hline & \multicolumn{2}{|c|}{ Languaje } & English & 44 \\
\hline & \multicolumn{2}{|c|}{ Criterio de Calidad } & $\begin{array}{l}\text { Revisión de experto en lectura detallada de cada } \\
\text { artículo, y su relación con el área de investigación. }\end{array}$ & 17 \\
\hline
\end{tabular}

Tabla 2 - Estructura Semántica de Búsqueda

Siguiendo la metodología, se aplican protocolos de revisión, que proporcionan resultados relevantes acorde a parámetros antes expuestos. En la sección de tipo de documento se ha seleccionado solamente artículos, considerando que se trata de trabajos resultantes de investigación, que abarcan todo el problema planteado. Finalmente, se realiza una lectura individual del resumen de cada artículo siguiendo lo expuesto en los criterios de calidad, para determinar su relación con la problemática planteada y las preguntas de investigación. 


\subsection{Preguntas de Investigación}

\subsubsection{RQ1: De los estándares que describen la UX, ¿Cuáles aplican en entornos educativos inclusivos?}

Es necesario conocer los estándares que describen la UX, así como la estructura del Sistema de Educación, para entender su funcionamiento, tipos, edades de aprendizaje e incluso conocer el tema de discapacidades, para poder relacionarlos.

\begin{tabular}{|c|c|c|}
\hline Variable & Indicador & Referencia Bibliográfica \\
\hline $\begin{array}{l}\text { ISO 9241- 11: Guía sobre } \\
\text { usabilidad }\end{array}$ & $\begin{array}{l}\text { Eficiencia, Efectividad, Seguridad, } \\
\text { Utilidad, Facilidad para recordar, } \\
\text { Facilidad de Aprendizaje }\end{array}$ & $\begin{array}{l}\text { (International Organization for } \\
\text { Standardization, 2018) }\end{array}$ \\
\hline $\begin{array}{l}\text { ISO 9241-210: Diseño } \\
\text { centrado en el ser } \\
\text { humano para sistemas } \\
\text { interactivos }\end{array}$ & $\begin{array}{l}\text { Experiencia de usuario, Satisfacción del } \\
\text { usuario, Accesibilidad, Percepciones, } \\
\text { Comportamientos }\end{array}$ & $\begin{array}{l}\text { (International Organization for } \\
\text { Standardization, 2010) }\end{array}$ \\
\hline \multirow{2}{*}{ Tipos de Educación } & Escolarizada o Formal & \multirow{2}{*}{ (UNESCO, 2009) } \\
\hline & No escolarizada o No Formal & \\
\hline \multirow{3}{*}{ Niveles de Educación } & Educación Inicial & \multirow{3}{*}{$\begin{array}{l}\text { (Ministerio de Educación de la } \\
\text { República del Ecuador, 2016) }\end{array}$} \\
\hline & Educación General Básica & \\
\hline & Bachillerato & \\
\hline \multirow{2}{*}{$\begin{array}{l}\text { Modalidades de } \\
\text { Educación }\end{array}$} & Presencial & \multirow{2}{*}{$\begin{array}{l}\text { (Ministerio de Educación de la } \\
\text { República del Ecuador, 2016) }\end{array}$} \\
\hline & Semipresencial, a distancia & \\
\hline \multirow{3}{*}{ Edades de aprendizaje } & De 4 a 5 años & \multirow{3}{*}{ (UNESCO - IBE, 2010) } \\
\hline & De 6 a 14 años & \\
\hline & De 15 a 17 años & \\
\hline Tipos de discapacidades & Física, sensorial, intelectual, psicológica & $\begin{array}{l}\text { (CONADIS - Ministerio De } \\
\text { Relaciones Laborales, 2013; Órgano } \\
\text { del Gobierno del Ecuador, 2012; } \\
\text { Siavichay Sinchi, 2016) }\end{array}$ \\
\hline
\end{tabular}

Tabla 3 - Estándares de la UX, que aplican en entornos educativos inclusivos

\subsubsection{RQ2: ¿Qué estrategias se han aplicado con sensores biom étricos para evaluar UAX?}

Se analiza las estrategias propuestas para trabajar con sensores biométricos (fisiológicos, biocinéticos y ambientales) y evaluar UAX. Conforme a las tendencias tecnológicas producidas por IoT, redes $5 \mathrm{G}$ e IA, es necesario conocer la forma de intervención que han aplicado en estudios previos. En ambientes inteligentes, estos sensores permiten evaluar comportamiento físico, fisiológico y emocional del usuario. 


\begin{tabular}{|c|c|c|}
\hline Variable & Indicador & Referencia Bibliográfica \\
\hline Sensores & Fisiológicos, Biocinéticos, Ambientales & (Bastidas \& Peláez, 2013) \\
\hline \multirow[b]{2}{*}{ Métricas } & $\begin{array}{l}\text { Tasa de rebote. Flujos de interacción. Ratio de } \\
\text { conversión }\end{array}$ & (Montero, 2015) \\
\hline & $\begin{array}{l}\text { Velocidad con la que el usuario realiza una tarea. } \\
\text { Cantidad de errores que comete. Frecuencia con que } \\
\text { rectifica el error. Cantidad de usuarios que completan } \\
\text { la tarea satisfactoriamente }\end{array}$ & $\begin{array}{l}\text { (Calvo-Fernández Rodríguez, } \\
\text { Ortega Santamaría, \& Valls } \\
\text { Saez, 2011) }\end{array}$ \\
\hline Tipo de análisis & Cualitativo y Cuantitativo & (Montero, 2015) \\
\hline
\end{tabular}

Tabla 4 - Métricas que se utiliza para evaluar UAX con sensores biométricos

\subsubsection{RQ3: ¿Cuáles son los métodos/instrumentos considerados para evaluar la UAX de personas con Síndrome de Down en ambientes educativos?}

Se detallan los métodos/instrumentos, herramientas y técnicas que son considerados según los tipos de desarrollo y aprendizaje para evaluar las emociones en las personas con Síndrome de Down. Los resultados de esta pregunta, permiten establecer criterios sustentables para la propuesta de proyectos de investigación.

\begin{tabular}{|c|c|c|}
\hline Variable & Indicador & Referencia Bibliográfica \\
\hline $\begin{array}{l}\text { Afecciones de la } \\
\text { trisomía } 21\end{array}$ & $\begin{array}{l}\text { Desarrollo cognitivo, Desarrollo } \\
\text { psicomotor, Desarrollo afectivo }\end{array}$ & (Marcelli \& Cohen, 2007) \\
\hline \multirow{12}{*}{ Emociones } & Asco & (Ekman, Paul \& Friesen, 1971) \\
\hline & Amor & (Norman, 2004; Parrott, 2001) \\
\hline & Ira & $\begin{array}{l}\text { (Ekman, Paul \& Friesen, 1971; Izard E., } \\
\text { 1972; Norman, 2004; Parrott, 2001) }\end{array}$ \\
\hline & Interés, culpa, desprecio, Vergüenza & (Izard E., 1972) \\
\hline & Disgusto & (Izard E., 1972; Plutchik, 2001) \\
\hline & Alegría & $\begin{array}{l}\text { (Ekman, Paul \& Friesen, 1971; Izard E., } \\
\text { 1972; Parrott, 2001; Plutchik, 2001) }\end{array}$ \\
\hline & Ansiedad & (Norman, 2004) \\
\hline & Enojo & $\begin{array}{l}\text { (Ekman, Paul \& Friesen, 1971; Plutchik, } \\
\text { 2001) }\end{array}$ \\
\hline & Miedo & $\begin{array}{l}\text { (Ekman, Paul \& Friesen, 1971; Izard E., } \\
\text { 1972; Norman, 2004; Parrott, 2001; } \\
\text { Plutchik, 2001) }\end{array}$ \\
\hline & Sorpresa & $\begin{array}{l}\text { (Ekman, Paul \& Friesen, 1971; Izard E., } \\
\text { 1972; Parrott, 2001; Plutchik, 2001) }\end{array}$ \\
\hline & Tristeza & $\begin{array}{l}\text { (Ekman, Paul \& Friesen, 1971; Parrott, } \\
\text { 2001; Plutchik, 2001) }\end{array}$ \\
\hline & Felicidad o satisfacción & $\begin{array}{l}\text { (Ekman, Paul \& Friesen, 1971; Norman, } \\
\text { 2004) }\end{array}$ \\
\hline
\end{tabular}




\begin{tabular}{|c|c|c|}
\hline Variable & Indicador & Referencia Bibliográfica \\
\hline \multirow{2}{*}{ Tipos de Educación } & Escolarizada o Formal & \multirow{2}{*}{ (UNESCO, 2009) } \\
\hline & No escolarizada o No Formal & \\
\hline \multirow{2}{*}{$\begin{array}{l}\text { Tipos de Capacidad } \\
\text { intelectual }\end{array}$} & Discapacidad intelectual & \multirow{2}{*}{ (Castillero Mimenza, 2019) } \\
\hline & Superdotación Intelectual & \\
\hline \multirow{5}{*}{$\begin{array}{l}\text { Métodos e } \\
\text { Instrumentos }\end{array}$} & Convolutional Neural Networks (CNN) & $\begin{array}{l}\text { (Ouherrou, Elhammoumi, } \\
\text { Benmarrakchi, \& El Kafi, 2019) }\end{array}$ \\
\hline & $\begin{array}{l}\text { Cuestionarios, Entrevistas, Indagación, } \\
\text { Observación }\end{array}$ & $\begin{array}{l}\text { (Hernández Sampieri, Collado } \\
\text { Fernández, \& Baptista Lucio, 2010) }\end{array}$ \\
\hline & Medición del esfuerzo mental & $\begin{array}{l}\text { (Ouherrou et al., 2019; P. Torres- } \\
\text { Carrion, 2017) }\end{array}$ \\
\hline & $\begin{array}{l}\text { Test, Prototipo y categorización, } \\
\text { Inspección }\end{array}$ & (Sutherland, 2013) \\
\hline & $\begin{array}{l}\text { LATCH-ON. Síndrome de Down: } \\
\text { Lectura y Escritura }\end{array}$ & $\begin{array}{l}\text { (Muro Haro, Santana Mancilla, \& } \\
\text { García Ruiz, 2012) }\end{array}$ \\
\hline \multirow[t]{2}{*}{ Herramientas } & $\begin{array}{l}\text { eScience. Realidad virtual. Simulación. } \\
\text { Modelado. Creación de prototipos } \\
\text { rápidos. PrEmo. AttrakDiff }\end{array}$ & (Córdoba-Cely, 2012) \\
\hline & Reejack & (Montero, 2015) \\
\hline Técnicas & $\begin{array}{l}\text { Card Sorting o agrupación de tarjetas. } \\
\text { Pruebas A/B }\end{array}$ & (Montero, 2015) \\
\hline
\end{tabular}

Tabla 5 - Métodos e instrumentos considerados en la UAX de personas con Síndrome de Down en ambientes educativos

\subsubsection{RQ4: ¿Qué características tienen los recursos didácticos que se han desarrollado para personas con discapacidades cognitivas?}

Conocer si existen recursos didácticos educativos desarrollados para entes con discapacidades cognitivas y cuáles son sus características, resulta fundamental para evaluar la relación del ámbito didáctico y variables de interacción de la UAX.

\begin{tabular}{lll}
\hline Variable & Indicador & Referencia Bibliográfica \\
\hline Tipos de recursos didácticos & $\begin{array}{l}\text { Auditivos, Gráficos, Imagen } \\
\text { Fija, Juegos, Impresos, TIC, } \\
\text { Mixtos: películas, videos, } \\
\text { tridimensionales. }\end{array}$ & $\begin{array}{l}\text { (Jiménez de la Cruz, 2016; Soto- } \\
\text { Ardila, Melo Niño, Caballero, \& } \\
\text { Luengo, 2019) }\end{array}$ \\
\hline Categorías de evaluación & $\begin{array}{l}\text { Atención, memoria auditiva, } \\
\text { memoria visual }\end{array}$ & $\begin{array}{l}\text { (Serna Jaramillo, Vanegas Uribe, } \\
\text { Álvarez Rueda, Niño Restrepo, \& } \\
\text { Ramírez Salazar, 2005). }\end{array}$ \\
\hline $\begin{array}{l}\text { Clasificación de los recursos } \\
\text { didácticos según etapas }\end{array}$ & Infantil, primaria, secundaria & (Akros, 2016) \\
\hline
\end{tabular}

Tabla 6 - Recursos didácticos educativos que se utilizan para trabajar con personas con discapacidades cognitivas 


\subsubsection{RQ5: ¿Qué estrategias se han utilizado en investigaciones conjuntas de UAX con IA?}

Conocer las estrategias en investigación, es dar un paso más allá de la metodología, y establecer desde la experiencia de los investigadores, como han logrado enlazar dos campos emergentes de la investigación científica (IA y UAX).

\begin{tabular}{lll}
\hline Variable & Indicador & Referencia Bibliográfica \\
\hline $\begin{array}{l}\text { Escenarios de } \begin{array}{l}\text { Inteligencia } \\
\text { Artificial }\end{array} \\
\text { Tecnologías de IA }\end{array}$ & $\begin{array}{l}\text { Inteligencia artificial estrecha } \\
\text { (Gomes \& Preto, 2018) }\end{array}$ \\
\cline { 2 - 2 } & Súper inteligencia artificial & $\begin{array}{l}\text { (Bizarro Torres, Luengo González, \& } \\
\text { Carvalho, 2018; Gomes \& Preto, 2018; } \\
\text { Jaramillo, Occhiuto, \& Garzotto, 2018; } \\
\text { Morphy, 2018) }\end{array}$ \\
\hline
\end{tabular}

Tabla 7 - Estrategias utilizadas en investigaciones conjuntas de UAX con IA

\subsection{Revisiones Sistemáticas Relacionadas}

$\mathrm{Al}$ realizar la búsqueda de SLR relacionados, aplicando el script general con filtrado orientado a "review", "slr", "meta análisis" o "survey", no se encontraron investigaciones publicadas sobre "Experiencia Afectiva de Usuario en ambientes con Inteligencia Artificial, Sensores Biométricos y/o Recursos Digitales Accesibles", menos aún relacionados con Síndrome de Down. Un trabajo cercano a la temática, se refiere a UX (Zaki, Wook, \& Ahmad, 2015), donde se concluye que para mejorar la capacidad cognitiva de personas que padecen discapacidades como Síndrome de Down, autismo y trastorno por déficit de atención e hiperactividad (TDAH), la mayoría de investigadores lo hacen usando un estímulo de aprendizaje mediante juegos serios e interacciones que incluyen realidad virtual (RV).

\subsection{Selección de revistas y Base de datos}

\subsubsection{Bases de Datos}

Se trabaja con la base de datos Scopus, por el prestigio y alcance que tiene en el ámbito de la ciencia, así como las herramientas y facilidades que permiten realizar una búsqueda rápida y eficiente. Como resultado de la búsqueda se generaron 44 artículos (ver Tabla 2), previa revisión de calidad que se realiza de forma cualitativa con ayuda de expertos.

\subsubsection{Listado de Revistas}

Los 44 artículos resultantes pertenecen a 37 revistas ("Listado de revistas," 2019). La revista de mayor relevancia es la de "Computers In Human Behavior", misma que cubre la interacción humano-computador y la ciberpsicología. Aborda el uso de computadoras en psicología, psiquiatría y disciplinas relacionadas, así como el impacto psicológico del 
uso de computadoras en individuos, grupos y la sociedad, además se publican trabajos teóricos originales, informes de investigación, revisiones de literatura, revisiones de software, reseñas de libros y anuncios (Guitton, 2019).

\section{Reporte de Revisión}

Una vez analizados los artículos resultantes, se ha organizado los resultados en función de la estructura de las variables en las preguntas de investigación.

\subsection{RQ1- De los estándares que describen la UX, ¿Cuáles aplican en entornos educativos inclusivos?}

Los estándares que describen la UX son: ISO 9241- 11 e ISO 9241-210, se enfocan en la usabilidad para alcanzar la facilidad de aprendizaje, así como la experiencia y satisfacción del usuario gracias al análisis de comportamientos y percepciones.

Los niños con discapacidades de aprendizaje (DA) muestran algunas dificultades emocionales y problemas de comportamiento en el aula, que conducen a la falta de motivación en el aprendizaje en comparación con sus compañeros sin DA (Ouherrou et al., 2019), pero gracias al uso de la Tecnología de la Información y la Comunicación (TIC) ha sido posible mejorar el aprendizaje colaborativo y social en clase, así como generar emociones positivas, como la atención y cooperación (Yoon \& Baek, 2018) en niños de quinto y sexto grado.

\subsection{RQ2 - ¿Qué estrategias se han aplicado con sensores biométricos para evaluar UAX?}

Los tipos de sensores más utilizados para evaluar la expresión de emociones son fisiológicos (presión arterial, medición de la glucosa en la sangre, electrocardiografía, electroencefalograma) y ambientales (humedad, luz, nivel de presión sonora, temperatura), que van ubicados en lugares estratégicos y sirven para evaluar la UX mediante las emociones. De forma estratégica (Sefidgar et al., 2016) basa su estudio en los éxitos de la terapia humano-animal (robot social -> criatura háptica), en una interacción diseñada para calmar o examinar la eficacia en aplicaciones terapéuticas como el manejo de la ansiedad; trabaja con una muestra de 38 mujeres entre 19-45 años de edad, a las que se conecta sensores fisiológicos. En la interacción, éstas sostienen el robot en sus regazos y lo acarician, dando como resultado la disminución de sus frecuencias cardíacas y respiratorias, identificándose como más tranquilas y felices. Los autores proponen el robot háptico, como una terapia con impacto emocional para ayudar a las personas con trastornos mentales a mejorar sus sentimientos de autoeficacia. De igual manera, haciendo uso de háptica efectiva, Eid \& Al Osman (2016) investigan el tacto afectivo, analizando cómo los participantes reaccionan emocionalmente a los estímulos y exploran la calidad de los métodos de medición de la experiencia como las reacciones fisiológicas (frecuencia cardíaca, expresiones faciales, actividad electrodérmica, expresiones gestuales o las actividades cerebrales), utilizando dos clases de hápticas afectivas: 1) juego / entretenimiento para hápticos afectivos, que se refiere a la utilización de juegos y entretenimiento para inducir comportamientos espontáneos, que conducen a una medición transparente de las emociones; 2) hápticos afectivos para 
juego / entretenimiento, que implican utilizar la modalidad háptica para mejorar la interactividad y la inmersión en juegos y entretenimiento al aumentar los contenidos audiovisuales existentes con interacciones físicas.

Doumbouya, Benlamine, Dufresne, \& Frasson (2018), detectan reacciones emocionales, utilizando medidas subjetivas a través del cuestionario y medidas objetivas a través de la actividad de las ondas cerebrales (electroencefalografía - EEG), combinadas con datos de seguimiento ocular y expresiones faciales para anotar los objetos del juego percibidos con las emociones dominantes expresadas. Para establecer la asociación entre los elementos del juego y la emoción dominante se utiliza el sistema de evaluación denominado EMOGRAPH (Gráfico emocional), que utiliza datos de seguimiento ocular y expresiones faciales.

Finalmente, los sensores ambientales que presenta (Berger et al., 2018), destacan su utilización con una herramienta de diseño como lo es Sensing Home, que permite a las personas integrar sensores y conectividad en productos domésticos de uso diario para que sean inteligentes, también pueden ser utilizados por personas para experimentar y explorar sensores en el hogar y en la vida diaria comprendiendo los problemas y las oportunidades de datos de los sensores desde sus hogares.

\subsection{RQ3 - ¿Cuáles son los métodos/instrumentos considerados para evaluar la UAX de personas con síndrome de Down en ambientes educativos?}

Los niños con problemas de aprendizaje, padecen de trastornos neurológicos que afectan sus capacidades cognitivas. Ouherrou, Elhammoumi, Benmarrakchi, \& El Kafi (2019) resaltan que las emociones constituyen una parte importante en el proceso de aprendizaje de los niños y es conveniente detectarlas a través de sus expresiones faciales. Esto se logra gracias a la evaluación de los niños con problemas de aprendizaje mediante un juego educativo que muestra algunas dificultades emocionales y problemas de conducta en el aula en comparación con sus compañeros. Además, con el sistema de reconocimiento de expresión facial basado en redes neuronales convolucionales, se analiza siete expresiones básicas de emociones faciales: enojo, asco, miedo, alegría, tristeza, sorpresa y neutro (Ekman, Paul \& Friesen, 1971), mismas que cada niño experimenta con un nivel diferente.

\subsection{RQ4 - ¿Qué características tienen los recursos didácticos que se han desarrollado para personas con discapacidades cognitivas?}

Los recursos didácticos se basan en el uso de la TIC, y juegos con diferentes contenidos multimedia como imágenes, sonidos, videos y animación (Ouherrou et al., 2019). Recursos que han sido desarrollados para personas con discapacidades cognitivas permitiendo mejorar su calidad de vida y eliminando las barreras en el aprendizaje, gracias al desarrollo de su afectividad, intelecto y motricidad, mediante la evaluación de su atención, memoria auditiva y visual. Cabe recalcar que las emociones y los estados afectivos influyen en la manera de pensar y la calidad del procesamiento de la información en el aprendizaje, principalmente en niños de primaria. 


\subsection{RQ5 - ¿Qué estrategias se han utilizado en investigaciones conjuntas de UAX con IA?}

Con la inclusión de nuevas tecnologías, la Experiencia de Usuario aumenta, considerando que la IA evoluciona día a día y está siendo utilizada para interactuar con el humano haciendo uso del computador, estimulando señales visuales, sonoras y mixtas, permitiendo detectar emociones en los niños mediante el análisis de sus expresiones faciales; además, los juegos serios pueden ser herramientas útiles para la enseñanza de estrategias de regulación emocional en adolescentes, desarrollando la capacidad de identificar y modificar las emociones muy intensas y la excitación durante los mismos (Vara et al., 2016).

La háptica afectiva ayuda a mejorar las competencias de niños con discapacidades cognitivas y reducir comportamientos estereotipados; misma que al interactuar con los robots, y estos emitir una respuesta emocional a través del tacto, salida de voz y gestual, pueden generar un efecto terapéutico positivo en los pacientes y ayudarlos a sentirse menos solitarios (Eid \& $\mathrm{Al}$ Osman, 2016). De igual forma, existe un tipo de robot animal diseñado específicamente para fines terapéuticos (Sefidgar et al., 2016), logrando efectos positivos sobre el estado de ánimo, comportamientos sociales e indicadores fisiológicos en los ancianos.

En el ámbito educativo, la realidad aumentada (AR) está siendo utilizada como un sistema de aprendizaje para niños pequeños, mejorando las habilidades espaciales y los factores de motivación (Han, Jo, Hyun, \& So, 2015); así mismo, la integración de los robots atrae la atención de los niños para el aprendizaje y por ende mejora sus resultados, promoviendo el conocimiento científico y técnico, así como las relaciones sociales, la imaginación, la sensibilidad artística y principalmente el aumento en las emociones positivas (Yoon \& Baek, 2018).

\section{Discusión, conclusiones y trabajos futuros}

La metodología de revisión sistemática de literatura ayuda a filtrar y organizar la información más relevante proporcionándonos datos exactos referentes a nuestra búsqueda por lo que pasamos a la discusión y conclusiones del estudio:

Los estándares ISO 9241-11 e ISO 9241-210 tienen mayor relación con la Experiencia de Usuario, ya que permiten evaluar los comportamientos y emociones provocadas como el enojo, asco, miedo, alegría, tristeza, sorpresa, disgusto y neutral. Cabe recalcar que, Ouherrou et al. (2019) resaltan que las emociones constituyen una parte importante en el proceso de aprendizaje de los niños y es conveniente detectarlas a través de sus expresiones faciales; además, de utilizar sensores fisiológicos y ambientales ya que pueden detectar emociones o estímulos internos y externos; así como, los robots hápticos (Sefidgar et al., 2016), convitíendose en estrategia válida en la relación UAX - IA.

Los pocos estudios realizados sobre personas con discapacidades intelectuales incluyeron niños de 6 a 14 años de edad, que reciben educación formal y utilizan los juegos y la interacción con la tecnología como principales recursos didácticos digitales, que permiten evaluar la Experiencia de Usuario y mejorar la atención, memoria auditiva y visual de los niños conforme lo indica Ouherrou et al.(2019). 
Para realizar pruebas de validación de UAX se utilizan herramientas de simulación que son idóneas para ensayar, conocer el funcionamiento de determinados sistemas o anticiparse a posibles problemas.

Al no encontrarse estudios específicos sobre "Experiencia Afectiva de Usuario en ambientes con Inteligencia Artificial, Sensores Biométricos y/o Recursos Digitales Accesibles”, para personas con Síndrome de Down, se abren las puertas para trabajos futuros como evaluar la UAX con IA Conversacional en alumnos con Síndrome de Down haciendo uso de la interacción gestual y robótica; también, validar métricas emergentes para la evaluación de experiencia afectiva de usuario con IA conversacional y sensores biométricos en entornos educativos universitarios.

\section{Referencias}

Akros (2016). 20 Recursos educativos para Educación especial. Retrieved from https:// akroseducational.es/blog/recursos-educativos-para-educacion-especial/

Bastidas, S. E. C., \& Peláez, J. M. L. (2013). Estudio de redes de sensores y aplicaciones orientadas a la recolección y análisis de señales biomédicas. Gerencia Tecnológica Informática, 12(33), 85-99. Retrieved from http://dialnet.unirioja.es/descarga/ articulo/4546834.pdf

Berger, A., Kurze, A., Totzauer, S., Storz, M., Lefeuvre, K., Bischof, A., \& Freiermuth, M. (2018). Sensing home: Designing an open tool that lets people collect and interpret simple sensor data from their homes. I-Com, 17(2), 153-167. https://doi. org/10.1515/icom-2018-0013

Bizarro Torres, N., Luengo González, R., \& Carvalho, J. L. (2018). Roamer, un robot en el aula de Educación Infantil para el desarrollo de nociones espaciales básicas. RISTI Revista Ibérica de Sistemas e Tecnologias de Informação, (28), 14-28. https://doi. org/10.17013/risti.28.14-28

Boada, N. (2017). ¿Por qué es tan importante el User Experience o Experiencia del Usuario? Retrieved March 13, 2019, from https://www.cyberclick.es/numericalblog/por-que-user-experience-o-experiencia-del-usuario

Brinquis, C. (2019). IA conversacional: conversaciones reales con un ordenador. Retrieved April 13, 2019, from https://www.incentro.com/es-es/blog/stories/aiconversacional-conversaciones-reales-con-un-ordenador/

Buck, R., Khan, M., Fagan, M., \& Coman, E. (2018). The User Affective Experience Scale: A Measure of Emotions Anticipated in Response to Pop-Up Computer Warnings. International Journal of Human-Computer Interaction, 34(1), 25-34. https://doi. org/10.1080/10447318.2017.1314612

Calvo-Fernández Rodríguez, A., Ortega Santamaría, S., \& Valls Saez, A. (2011). Métodos de evaluación con usuarios. Retrieved from http://openaccess.uoc.edu/webapps/ o2/bitstream/10609/9861/4/PID_o0176614.pdf 
Castells, S., \& Wisniewski, K. E. (1994). Growth hormone treatment in Down syndrome. Journal of Pediatrics, 124, 158-159. https://doi.org/10.1016/Soo223476(94)70277-2

Castillero Mimenza, O. (2019). ¿Qué es el Cociente Intelectual (CI)? Retrieved May 27, 2019, from https://psicologiaymente.com/inteligencia/cociente-intelectual

CONADIS - Ministerio De Relaciones Laborales. (2013). Manual De Buenas Prácticas Para La Inclusión Laboral De Personas Con Discapacidad (p. 40). p. 40. Retrieved from http://www.trabajo.gob.ec/wp-content/uploads/downloads/2013/12/ MANUALFIN.pdf

Córdoba-Cely, C. (2012). La experiencia de usuario: de la utilidad al efecto. La Experiencia de Usuario: De La Utilidad Al Efecto. Iconofacto, 9(1), 56-70. Retrieved from https://revistas.upb.edu.co/index.php/iconofacto/article/view/1919

Desmet, P., \& Hekkert, P. (2007). Framework of Product Experience Human-Product Interaction. International Journal of Design, 1(1), 57-66. https://doi.org/10.1210/ en.2017-00348

Doumbouya, R., Benlamine, M. S., Dufresne, A., \& Frasson, C. (2018). Game scenes evaluation and player's dominant emotion prediction. pp. 54-65. https://doi. org/10.1007/978-3-319-91464-0_6

Eid, M. A., \& Al Osman, H. (2016). Affective Haptics: Current Research and Future Directions. IEEE Access, 4, 26-40. https://doi.org/10.1109/ACCESS.2015.2497316

Ekman, P., \& Friesen, W. V. (1971). Universals and cultural differences in facial expressions of emotion - Ekman \& Friesen.pdf. Journal of Personality and Social Psychology, 19, 208-282. Retrieved from https://es.scribd.com/doc/308255392/ Universals-and-Cultural-Differences-in-Facial-Expressions-of-Emotion-PaulEkman\#logout

Florez, J. (2004). Patologia cerebral y sus repercusiones cognitivas en el Sindrome de Down. Retrieved from http://www.downcantabria.com/articuloN1.htm

García, A. (2012). Inteligencia artificial : fundamentos, práctica y aplicaciones. Retrieved from https://www.academia.edu/20419747/INTELIGENCIA_ARTIFICIAL_ Fundamentos_práctica_y_aplicaciones

Gomes, C. C., \& Preto, S. (2018). Artificial intelligence and interaction design for a positive emotional user experience. Advances in Intelligent Systems and Computing, 722, 62-68. https://doi.org/10.1007/978-3-319-73888-8_11

Guitton, M. (2019). Computers in Human Behavior. Retrieved from https://www. journals.elsevier.com/computers-in-human-behavior/

Han, J., Jo, M., Hyun, E., \& So, H.-J. (2015). Examining young children's perception toward augmented reality-infused dramatic play. Educational Technology Research and Development, 63(3), 455-474. https://doi.org/10.1007/s11423-015-9374-9 
Hernández Sampieri, R., Collado Fernández, C., \& Baptista Lucio, M. del P. (2010). Metodología de la Investigación (5ta ed.). Retrieved from https://www.academia. edu/23889615/._Hernández_Sampieri_R._Fernández_Collado_C._y_Baptista_ Lucio_M._P_2010_?auto=download

Herrero Nivela, M. L., Vived Conte, E., \& Digitalia (2007). Programa de comprensión, recuerdo y narración : una herramienta didáctica para la elaboración de adaptaciones curriculares : experiencia en alumnos con síndrome de Down. Retrieved from https://books.google.com.ec/books?id=b-3vzdDhMRMC\&pg=PA16\&lpg=PA16\&d $\mathrm{q}=$ percepcion+sindrome+de+down+florez+1994\&source=bl\&ots=pGNEgliWYo\&s ig=ACfU3U2gBoJ3LjC_GC8B-3b434EIuqhKfg\&hl=es\&sa=X\&ved=2ahUKEwilmP OV8rnkAhXqs1kKHSamBmoQ6AEwEHoECAkQAQ\#v=onepage\&q=percep

International Organization for Standardization (2010). ISO 9241-210 Ergonomics of human-system interaction-Human-centred design for interactive systems. Retrieved from https://www.sis.se/api/document/preview/912053/

International Organization for Standardization (2018). ISO 9241-11:2018 - Ergonomics of human-system interaction -- Part 11: Usability: Definitions and concepts. Retrieved May 4, 2019, from https://www.iso.org/standard/63500.html

Izard E., C. (1972). Patterns of emotions; a new analysis of anxiety and depression by Carroll E. Izard. Retrieved from https://catalogue.nla.gov.au/Record/446907

Jaramillo, J.C., Occhiuto, D., \& Garzotto, F. (2018). Artworks' Features DiscoveryThrough Engaging Conversations for Children. IOP Conference Series: Materials Science and Engineering, 364(1). https://doi.org/10.1088/1757-899X/364/1/012097

Jiménez de la Cruz, C. A. (2016). Tipos de Recursos Didácticos - Recursos Didácticos y Tenologicos. Retrieved May 4, 2019, from https://sites.google.com/site/ recursosdidacticosytenologicos/tipos-de-recursos-didacticos

Kitchenham, B., Brereton, O. P., Budgen, D., Turner, M., Bailey, J., \& Linkman, S. (2009). Systematic literature reviews in software engineering - A systematic literature review. Information and Software Technology, 51(1), 7-15. https://doi. org/10.1016/j.infsof.2008.09.009

Listado de revistas (2019). Listado de revistas. Retrieved from https://drive.google. com/file/d/1Ac3sVNnjBEh9ZLHEmEWGTldm8gkVXo4f/view?usp=sharing

Marcelli, D., \& Cohen, D. (2007). Manual de Psicopatología del niño (7ma ed.). Retrieved from https://www.casadellibro.com/libro-manual-de-psicopatologia-del-nino-7ed/9788445817865/1156313

Marcos, M.-C. (2001). HCI (Human Computer Interaction): Concepto Y Desarrollo. El Profesional de La Informacion, 10(6), 4-16. Retrieved from https://www. academia.edu/308723/HCI_Human_Computer_Interaction_Concepto_Y_ Desarrollo?auto=download 
Ministerio de Educación de la República del Ecuador. (2016). Ley Orgánica de Educación Intercultural. (p. 102). Retrieved from https://www.google.com/ url sa $=t \& r c t=j \& q=\& e s r c=s \&$ source $=$ web\&cd $=1 \& c a d=r j a \& u a c t=8 \& v e d=2 a h$ UKEwixp9_T $88 \mathrm{HmAhVCbKwKHQXGBBAQFjAAegQIAhAC \& url=}$ https\%3A\%2F\%2Feducacion.gob.ec\%2Fwp-content\%2Fuploads\%2Fdownloads\%2 F2017\%2Fo5\%2FLey-Organica- Educacion-Intercultural-Codif

Mira, J. (1999). Inteligencia artificial, emoción y neurociencia. Arbor, 162(640), 473506. https://doi.org/10.3989/arbor.1999.i640.1656

Montero, Y. H. (2015). Experiencia de Usuario: Principios y Métodos. Retrieved from https://yusef.es/Experiencia_de_Usuario.pdf

Morphy, E. (2018). What Is Conversational User Experience (UX). Retrieved June 27, 2019, from https://www.cmswire.com/digital-experience/what-is-conversationaluser-experience-ux/

Muro Haro, B. P., Santana Mancilla, P. C., \& García Ruiz, M. Á. (2012). Uso de interfaces tangibles en la enseñanza de lectura a niños con síndrome de Down. El Hombre y La Máquina, (25). Retrieved from https://www.redalyc.org/articulo. oa?id=47824590004

Norman, D. A. (2004). Emotional Design. The Design Of Everyday Things. In Emotional Design. The Design Of Everyday Things, 13, 67-80. https://doi.org/http://dx.doi. org/10.1300/Joo7v13no2_06

Órgano del Gobierno del Ecuador (2012). Ley Orgánica de Discapacidades. Cuadernos de Derecho Público, 47-78. Retrieved from https://www.consejodiscapacidades. gob.ec/wp-content/uploads/downloads/2014/02/ley_organica_discapacidades. pdf

Ouherrou, N., Elhammoumi, O., Benmarrakchi, F., \& El Kafi, J. (2019). Comparative study on emotions analysis from facial expressions in children with and without learning disabilities in virtual learning environment. Education and Information Technologies, 24(2), 1777-1792. https://doi.org/10.1007/s10639-018-09852-5

Parrott, W. G. (2001). Emotions in Social Psychology: Essential Readings. In Ann Arbor: Edwards. Retrieved from https://books.google.com.ec/books?hl=es\&lr= \&id=jV5QVgM6Me8C\&oi $=$ fnd\&pg = PR11\&dq=Parrott, +W .+G.+(2001) .+ Emotions+in+Social+Psychology:+Essential+Readings.+Ann+Arbor:+Edwards.+ Psychology+Press.\&ots=05n5wSo44P\&sig =xZm1iMsQ7rFVsnHGZQ7mw4g2xWo

Plutchik, R. (2001). The Nature of Emotions. International Journal of Philosophical Studies, 27(1), 103-106. https://doi.org/10.1080/09672559.2019.1563979

Rondal, J., Perera, J., \& Nadel, L. (2000). Síndrome de Down : revisión de los últimos conocimientos. In ISBN 84-239-8997-6, págs. 167-184. Retrieved from https:// www.todocoleccion.net/libros-segunda-mano-medicina/sindrome-down-revisionultimos-conocimientos-coordinado-por-jean-rondal-juan-perera-y x152216762 
Sears, A., \& Jacko, J. A. (2007). Human-Computer Interaction: Fundamentals. Retrieved from http://citeseerx.ist.psu.edu/viewdoc/download;jsessionid= 92992AAoF69F7AA52C84EC732A44028C?doi=10.1.1.458.7291\&rep=rep1 \&type $=$ pdf

Sefidgar, Y. S., MacLean, K. E., Yohanan, S., Van Der Loos, H. F. M., Croft, E. A., \& Garland, E. J. (2016). Design and Evaluation of a Touch-Centered Calming Interaction with a Social Robot. IEEE Transactions on Affective Computing, 7(2), 108-121. https://doi.org/10.1109/TAFFC.2015.2457893

Serna Jaramillo, A. J., Vanegas Uribe, A. I., Álvarez Rueda, E. M., Niño Restrepo, N., \& Ramírez Salazar, D. A. (2005). Propuesta didáctica para el desarrollo de repertorios básicos de atención y memoria en niñas y niños con Síndrome de Down integrados al aula regular. Retrieved from http://tesis.udea.edu.co/bitstream/10495/3076/1/ SernaAdriana_2005_PropuestaDidacticaDesarrollo.pdf

Siavichay Sinchi, Y. T. (2016). Desarrollo social de las personas con discapacidad en Ecuador (Cuenca). Retrieved from http://dspace.ucuenca.edu.ec/ handle/123456789/23532

Soto-Ardila, L. M., Melo Niño, L., Caballero, A., \& Luengo, R. (2019). Estudio de las opiniones de los futuros maestros sobre el uso de los videojuegos como recurso didáctico a través de un análisis cualitativo. RISTI - Revista Ibérica de Sistemas e Tecnologias de Informação, (33), 48-63. https://doi.org/10.17013/risti.33.

Sutherland, I. (2013). Capítulo 2 Realidad virtual HCI (pp. 17-21). Retrieved from http:// catarina.udlap.mx/u_dl_a/tales/documentos/mcc/vera_p_le/capitulo2.pdf

Tesauro científico (2019). Tesauro científico. Retrieved from https://drive.google.com/ file/d/1AZDViS6eAYWhxR7MZaNrTUOl3tiok_05/view?usp=sharing

Thesaurus.com (2019). Synonyms and Antonyms of Words at Thesaurus.com. Retrieved from https://www.thesaurus.com/

Tolosa, C., \& Giz, Á. (2010). Sistemas Biométricos. Retrieved from https://www.dsi. uclm.es/personal/MiguelFGraciani/mikicurri/Docencia/Bioinformatica/web_ BIO/Documentacion/Trabajos/Biometria/Trabajo Biometria.pdf

Torres-Carrion, P. (2017). Evaluación de estrategias de aprendizaje con HCI kinect en alumnos con Síndrome Down. Retrieved from http://e-spacio.uned.es/fez/eserv/ tesisuned:ED-Pg-CyEED-Pvtorres/TORRES_CARRION_PabloVicente_Tesis.pdf

Torres-Carrión, P., González-González, C., Bernal-Bravo, C., \& Infante-Moro, A. (2019). Gesture-based children computer interaction for inclusive education: A systematic literature review. Communications in Computer and Information Science, 895, 133-147. https://doi.org/10.1007/978-3-030-05532-5_10

Torres-Carrion, P. V., Gonzalez-Gonzalez, C. S., Aciar, S., \& Rodriguez-Morales, G. (2018). Methodology for systematic literature review applied to engineering and education. In IEEE Global Engineering Education Conference, EDUCON, 2018-April(April), 1364-1373. https://doi.org/10.1109/EDUCON.2018.8363388 
UNESCO (2009). Policy Guidelines on Inclusion in Education. 38. Retrieved from https://unesdoc.unesco.org/ark:/48223/pfoooo177849

UNESCO - IBE (2010). World Data on Education Données mondiales de l' éducation Datos Mundiales de Educación. 7a edición, 54. Retrieved from http://www.ibe. unesco.org/

Vara, M. D., Baños, R. M., Rasal, P., Rodríguez, A., Rey, B., Wrzesien, M., \& Alcañiz, M. (2016). A game for emotional regulation in adolescents: The (body) interface device matters. Computers in Human Behavior, 57, 267-273. https://doi.org/10.1016/j. chb.2015.12.033

Yoon, M.-B., \& Baek, J.-E. (2018). Development and application of the STEAM education program based on the soccer robot for elementary students. International Journal of Mobile and Blended Learning, 10(3), 11-22. https://doi.org/10.4018/ IJMBL.2018070102

Zaki, N. A. A., Wook, T. S. M. T., \& Ahmad, K. (2015). Analysis and classification of serious games for cognitive stimulation. In Proceedings - 5th International Conference on Electrical Engineering and Informatics: Bridging the Knowledge between Academic, Industry, and Community, ICEEI 2015, 612-617. https://doi. org/10.1109/ICEEI.2015.7352572 